\title{
Post-traumatic Hemothorax: Management in a Limited-medium Structure
}

\author{
Jonatana $\mathrm{AD}^{1}$, Razafindraibe $\mathrm{VB}^{1}$, Rahantasoa Finaritra $\mathrm{CP}^{2}$, Randrianambinina $\mathrm{F}^{3}$, Samison \\ $\mathrm{LH}^{2}$, Rakotoarijaona $\mathrm{AH}^{1}$, Rakotovao $\mathrm{HJL}^{3}$ \\ *Correspondence: rcasimirfleurprudence@gmail.com / Tel: +261341900481 \\ ${ }^{1}$ Centre Hospitalier Universitaire Analankininina Toamasina Madagascar, General Surgery \\ Department \\ ${ }^{2}$ Joseph Ravoahangy Andrianavalona University Hospital Center Antananarivo Madagascar, \\ Visceral Surgery Service \\ ${ }^{3}$ Joseph Ravoahangy Andrianavalona University Hospital Center Antananarivo Madagascar, \\ Thoracic Surgery DepartmentAuteur
}

\begin{abstract}
Post-traumatic hemothorax is common in young adults in the context of road accidents. Explorations of the lesions may be limited by the technical platform. The objective of our study is to discuss the difficulty of managing post-traumatic hemothorax in a limited-medium structure. This was a 20-month retrospective study (01 May 2018-31 December 2019), involving 38 patients treated for post-traumatic hemothorax, whether or not associated with pneumothorax, in the general surgery department of the CHU Analankinina Toamasina. The hospital frequency was $1.66 \%$, with a mean age of the patients of 31.89 years, and a male predominance $(73.68 \%)$. The main provider of hemothorax was the civil liability accident (52.63\%). Chest X-rays were performed in almost all patients (94.74\%). Complications had been found in 4 patients with secondary surgery, with favorable outcomes. The frequency of hemothorax is constantly increasing in Madagascar, where the civil liability accident is the main cause. Chest X-ray remains the most accessible examination to be carried out in emergency. An early and efficient thoracic drainage generally ensures a favorable evolution without after-effects.
\end{abstract}

Keywords: Hemothorax, Trauma, Madagascar

Received : October 17, 2020

Received in Revised: October 25, 2020

Accepted: October 28, 2020

\section{Introduction}

More than half of all chest traumas can be complicated by hemothorax. The etiological circumstances are diverse and vary from country to country. In Africa, with the prevailing political and socio-economic context, chest wounds are also common, particularly stab wounds, which account for up to $80 \%$ of such wounds. In Madagascar, the frequency of posttraumatic hemothorax increases proportionally with the resurgence of violence and crime in the country. the country (Razafimanjato et al, 2020). The prognosis depends on the nature of the lesions and the precocity of management. This period of treatment can be extended when medical imaging is defective. The objective of our study is to discuss the difficulty of managing post-traumatic hemothorax in a limited-medium structure. 


\section{Methods}

This was a 20-month retrospective study, from May 1, 2018 to December 31, 2019, of the medical records of patients hospitalized and managed for post-traumatic hemothorax

or hemopneumothorax in the general surgery department of the CHU Analankininina Toamasina.

In this study, we included all records of patients hospitalized for post-traumatic hemothorax. Hemothoraxes of non-traumatic etiology were not included. The variables studied were: age, sex, occupation, type of trauma, etiologic circumstance, imaging examinations, type and abundance of hemothorax, associated lesions, treatment, evolution, length of stay.

\section{Results and Discussion}

During the study period, we recorded 38 cases of post-traumatic hemothorax with or without pneumothorax out of a total of 2286 hospitalized patients, i.e. a hospital frequency of $1.66 \%$.

The mean age was 31.89 years, with extremes ranging from 17 to 51 years. Male predominance was found with a proportion of $73.68 \%(n=28)$, against $26.32 \%(n=10)$ for the female gender. The sex ratio was 2.8. People without a permanent job were the most affected with 22 cases, or $57.89 \%$ (Table 1). The traumatic context most often encountered was a civil liability accident (CLA) in 52.63\% ( $\mathrm{n}=20)$. Road traffic accidents (MVAs) accounted for $31.58 \%$ of cases.

Table 1. Breakdown by occupation

\begin{tabular}{|l|c|c|}
\hline Profession & Headcount (n) & Rate (\%) \\
\hline Unemployed & 22 & 57.89 \\
\hline Civil servant & 8 & 21.05 \\
\hline Worker & 2 & 5.26 \\
\hline Student & 4 & 10.53 \\
\hline Driver & 2 & 5.26 \\
\hline Total & 38 & 100 \\
\hline
\end{tabular}

The type of trauma was a closed chest trauma in more than half of the cases, $57.89 \%(n=22)$, compared to $42.11 \%(\mathrm{n}=16)$ for a penetrating chest wound. The agent responsible for the penetrating wound was a knife in $75 \%(\mathrm{n}=12)$ and a firearm in $25 \%(\mathrm{n}=4)$. The wound site was supra-nipple in the majority of cases, $62.50 \%(n=10)$, compared to $37.50 \%(n=6)$ in the sub-nipple. Chest X-ray was the most common imaging examination, accounting for $94.74 \%$ $(n=36)$ of cases (Table 2). A very abundant hemothorax was found in almost half of our patients, 47.37\% $(\mathrm{n}=18)$. Low-abundance hemothoraxes, generally requiring only simple monitoring, were $15.79 \%(\mathrm{n}=6)$ (Table 3$)$. Hemothorax was associated with pneumothorax in 12 other patients $(31.58 \%)$.

Table 2. Imaging examinations performed

\begin{tabular}{|l|c|c|}
\hline Imaging & Headcount (n) & Rate (\%) \\
\hline Chest X-ray & 36 & 94.74 \\
\hline Chest Scanner & 4 & 10.53 \\
\hline Chest ultrasound & 2 & 5.26 \\
\hline
\end{tabular}


Table 3. Quantity of effusion

\begin{tabular}{|l|c|c|}
\hline Spreads & Headcount $(\mathrm{n})$ & Rate $(\%)$ \\
\hline Low abundance & 6 & 15.79 \\
\hline Medium abundance & 14 & 36.84 \\
\hline Great abundance & 18 & 47.37 \\
\hline Total & 38 & 100 \\
\hline
\end{tabular}

Limb trauma were the most frequently observed associated lesions at 50\% (n=19). The association of costal fractures was found in 10 patients $(26.32 \%)$, while no associated intraabdominal lesions were found (Table 4). Thoracic drainage was performed in 32 patients $(84.21 \%)$, while simple monitoring was sufficient for 6 patients $(15.79 \%)$. Only 4 patients $(10.53 \%)$ had undergone surgery

Table 4. Associated lesions

\begin{tabular}{|l|c|c|}
\hline Lesions & Headcount $(\mathrm{n})$ & Rate $(\%)$ \\
\hline Costal fractures & 10 & 26.32 \\
\hline Limb injuries & 19 & 50 \\
\hline Craniofacial lesions & 8 & 21.05 \\
\hline Abdominal lesions & 0 & 0 \\
\hline Cervical lesions & 4 & 10.53 \\
\hline
\end{tabular}

for hemostasis $(n=2 ; 50 \%)$ of intercostal vascular wounds, and for management of infectious complications $(\mathrm{n}=2 ; 50 \%)$ such as septic shock and pachypleuritis on pyothorax. The approach used was posterolateral thoracotomy in $100 \%$ of cases, and the different procedures performed were 02 hemostasis thoracotomies, 01 thoracostomy, and 01 decortication. The postoperative evolution was favorable in $100 \%$ of the cases. The average length of hospitalization was 7.47 days, with extremes ranging from 3 to 27 days.

Traumatic etiology is the main cause of hemothorax. It is reported that 40 to $60 \%$ of chest trauma is complicated by hemothorax (Camara et al, 2014). The frequency of these posttraumatic hemothoraxes varies from one country to another, but is constantly increasing, especially in Madagascar as in most African countries, due to socio-economic factors, political instability and the resurgence of local violence and crime. Moreover, this increase is also linked to the increase in the frequency of public road accidents (Camara et al, 2014; Razafimanjato et al, 2020). In two studies carried out 6 years apart in the thoracic surgery department of Donka hospital in Guinea, the hospital frequency increased from $3.33 \%$ in 2008 (Barry, 2006) to 9.25\% in 2014 (Camara et al, 2014). In our series, the low hospital frequency found $(1.66 \%)$, is only explained by the fact that our service is a general surgery service, taking care of several other non-thoracic surgical pathologies, and that our center is the only reference center in the eastern region of Madagascar for visceral, pediatric and thoracic surgical pathologies. The average age of our patients was 31.89 years, with extremes ranging from 17 to 51 years. These figures are quite similar to those found by several African authors (Camara et al, 2014; Djigne, 2006), reflecting the predilection for chest trauma in young adults, with an observed tendency towards violence and risk behaviors such as hyperactivity, aggressiveness, contact sports, car racing, etc.

Male dominance is widely reported in the literature (Camara et al, 2014; Rajaonera et al, 2016). It is also observed in our study, with a sex ratio of 2.8. Indeed, the more violent and aggressive man favours traumatic pathologies. Moreover, in Africa, the majority of men are 
manual workers, with a high risk of occupational accidents. In the series of Camara et al, in Guinea, $37.84 \%$ of patients were workers (Camara et al, 2014).

The occupational categories most affected, however, vary according to the authors (Camara et al, 2014; Razafimanjato et al, 2020; Djigne, 2006). In our series, the category "without a permanent job" was the most represented with $57.89 \%$. This phenomenon is explained by the high unemployment rate observed in Madagascar, associated with the low schooling rate of the general population.

These different factors are more marked in the provinces, particularly in Toamasina, and are The creation of informal work, which is a source of socio-economic insecurity reflected in high levels of local crime. Faced with this crime, the judicial system is overwhelmed, and a loss of confidence in the justice system can be observed among the local population, with a high proportion of popular vindictiveness. Thus, CRA was the most frequent etiological circumstance in our study, with $52.63 \%$, followed by MVA with $31.58 \%$. According to several authors, MVA represents the main provider of post-traumatic hemothorax (Camara et al, 2014; Hama et al, 2017).

In our series, closed chest trauma was more frequent than penetrating wounds. These results are comparable to those found in the literature (Hama et al, 2017). In Africa, stab wounds are the most frequent thoracic wounds, with a proportion of up to $80 \%$ (Cador \& Lonjon, 1997). Ouadnouni et al. even reported a proportion of $98 \%$ across all chest wounds (Ouadnouni et al, 2014). These results are similar to those found by our team with a proportion of $75 \%$.

Chest X-rays were the most frequently performed imaging examination in our study $(94.74 \%$ of cases). It is the first-line examination for thoracic pathology, easy to use and with a high degree of accuracy. of realization, easy to access, and not expensive. It allows the diagnosis of hemothorax or hemopneumothorax to be made, an initial assessment of the associated lesions (costal, parenchymal, mediastinal, etc.), and the prioritization of other paraclinical examinations (Razafimanjato et al, 2020). For minimal or encysted effusions, pleural ultrasound is more indicated, with better sensitivity and specificity. It also has the advantage of being simple, fast, and non-invasive, while being within the reach of the Malagasy population. The scanner on the other hand is expensive, and is prescribed only occasionally, given the severity of the initial clinical picture and the appearance of progressive complications. It is important to emphasize that paraclinical examinations should in no case delay an urgent therapeutic procedure (Ouadnouni et al, 2014).

The association with a pneumothorax is quite frequent, especially in the case of penetrating trauma. $80 \%$ of these traumas are associated with a hemopneumothorax (Desjardins, 1998). In our series, $31.58 \%$ of patients had a hemopneumothorax. Post-traumatic hemothoraxes often occur in the context of multiple traumas or polytrauma. Different authors report that limb trauma is the most common injury.

\section{Conclusion}

In Africa, the frequency of post-traumatic hemothorax is constantly increasing. In the eastern region of Madagascar, the predominant traumatic etiology is ARC, with a high proportion of penetrating wounds, especially those caused by stabbing. This phenomenon is linked to the resurgence of local crime, with growing insecurity against a backdrop of political instability. The diagnosis of hemothorax is usually obtained by Chest X-ray, which is a test accessible to the population and available in all hospitals on the island. Early management of any posttraumatic hemothorax is recommended to ensure a favorable evolution without 
complications. Effective chest drainage combined with medical treatment is sufficient in the majority of cases to obtain a cure, without after-effects.

\section{References}

Camara AK, Magassouba AS, Naby CS, Sara A, Koghomou NA, \& Barry FM. (2014). Prise en charge des hémothorax post-traumatiques: une expérience unicentrique de l'hôpital national Donka. Med Afr Noire, 64(8/9) : 429-34.

Razafimanjato NNM, Tsiambanizafy GO, \& Ravoatrarilandy M (2020). Aspects chirurgicaux des plaies thoraciques pénétrantes par arme blanche dans pays à ressources limitées: A propos de 73 cas. Archives of the Balkan Médical Union, 55(1) : 55-63.

Ouadnouni Y, Ghalimi J, Lakranbi M, \& Smahi M. (2014). Les plaies thoraciques. J Maroc Sci Med, 19(1) : 17-22.

Barry FM. (2006). Traumatismes fermés du thorax : épidémiologie et traitement au service de chirurgie thoracique de l'Hôpital National Donka. [Thèse] de Doctorat en Médecine, $U G A N C$.

Djigne TA. (2006). Hémothorax post-traumatique: fréquence, prise en charge et complication au service de chirurgie viscérale de l'Hôpital National Donka. [Thèse] de Doctorat en Médecine, UGANC.

Rajaonera AT, Razafimanjato NNM, Raoelijaona LH, Rakotoarisoa AJC, Ravalisoa AML, Rakotovao HJL. (2016) Traumatismes Thoraciques : un défi de prise en charge en réanimation. Batna J Med Sci, 3, 108-11.

Hama Y, Rabiou S, Kabi I, Chaibou MS, Sani R, \& Harouna YD. (2017). Gestion des traumatismes thoraciques en milieu non spécialisé: quels résultats? JCTCV, 21(2) : 9.

Cador L, \& Lonjon T. (1997). Plaies thoraciques. Encycl Med Chir, Urgences. Paris : Elsevier Masson, 24(103) : 10-9.

Desjardins G. (1998). Les traumatismes pénétrants du thorax. Conférences d'actualisation 1998. 40e Congrès National Anesth Réa. SFAR. Paris : Elsevier; 615-24. 